\title{
Comments on "Interval type-2 fuzzy sets are generalization of interval-valued fuzzy sets: towards a wide view on their relationship" [2]
}

\author{
Jerry M. Mendel, Life Fellow, IEEE, Hani Hagras, Fellow, IEEE, Humberto Bustince, Member, IEEE \\ and Francisco Herrera, Member, IEEE
}

\begin{abstract}
This Letter makes some observations about [2] that further support the distinction between an interval type-2 fuzzy set (IT2 FS) and an interval-valued fuzzy set (IV FS), points out that all operations, methods and systems that have been developed and published about IT2 FSs are, so far, only valid in the special case when IT2 FS = IVFS, and suggests some research opportunities.
\end{abstract}

Index Terms-Interval type-2 fuzzy sets, interval valued fuzzy sets, type-2 fuzzy sets

$\mathrm{T}$ YPE-2 fuzzy sets (T2 FSs) have been beset with some unfortunate notation, although, as is explained in [9], this has not prevented their use nor has it led to any theoretically incorrect results. The work presented in [1] addressed the many facets of the notational problems and there are many useful recommendations in [1] to remedy them. Undoubtedly, the statement $J_{x} \subseteq[0,1]$ in the widely used definition of a T2 FS $\tilde{A}$ [7]

$$
\tilde{A}=\left\{\left((x, u), \mu_{\tilde{A}}(x, u)\right) \mid \forall x \in X, \forall u \in J_{x} \subseteq[0,1]\right\},
$$

has caused much confusion.

Recall that $J_{x} \subseteq[0,1]$ means: "every element of $J_{x}$ is also an element of $[0,1]$." Unfortunately, no formulaic definition was given in [7] for $J_{x}$ something thought to have been remedied in 2006 [8], where the following formula is given for $J_{x}$ :

$$
J_{x}=\left\{(x, u): u \in\left[\underline{\mu}_{\tilde{A}}(x), \bar{\mu}_{\tilde{A}}(x)\right]\right\}
$$

Submitted on February 11, 2015.

Jerry M. Mendel is with the Signal and Image Processing Institute, University of Southern California, Los Angeles California 90089-2564, USA (e-mail: mendel@sipi.usc.edu).

Hani Hagras is with the Computational Intelligence Centre, School of Computer Science and Electronic Engineering, University of Essex, Wivenhoe Park, Colchester, CO43SQ, United Kingdom (e-mail: hani@essex.ac.uk).

Humberto Bustince is with the Departamento de Automatica y Computacion, Universidad Publica de Navarra, Pamplona, 31006, Spain (email: bustince@unavarra.es).

Francisco Herrera is with the Departament of Computer Science and Artificial Intelligence, University of Granada, 18071 Granada, Spain (e-mail: herrera@decsai.ugr.es). in which $\mu_{\tilde{A}}(x)$ and $\bar{\mu}_{\tilde{A}}(x)$ are the lower and upper membership functions of $\tilde{A}$, respectively. The intent of [8] was to provide authors with standard background material about interval type-2 fuzzy logic systems that they could use, as is, for background materials in their papers, so that they would not have to re-invent such background materials. However, [8] is not widely known or used, because it is only found as a Standard on the website of the IEEE Computational Intelligence Society.

In [2], four different choices (examples) are given for $J_{x}$ which conform to the general meaning of $J_{x} \subseteq[0,1]$, namely: multisets, interval-valued fuzzy set (IVFS), multi-IVFS and combinations of numbers and intervals. Only one of these choices corresponds to the way $J_{x}$ has been used by the IT2 FS community, namely that of an interval-valued fuzzy set (IVFS). Consequently, [2] points out that the phrase "IT2 FS" is a more general term than the phrase "IVFS" and includes IVFS as a special case.

Although $J_{x}$ has not been ambiguous to the IT2 FS community since 2006 [8], and was understood by that community to mean (2), it is important to acknowledge that new researchers may become familiar with the T2 literature at different entry time points for them (e.g., the most common seems to be by reading [7]). One cannot expect them to imagine that there are notational problems. Additionally, it is no longer possible for new researchers to be knowledgeable about the very large T2 literature; so, since $J_{x} \subseteq[0,1]$ has been used from the very beginning one must abide by the general meaning of $J_{x} \subseteq[0,1]$.

As acknowledged by Nilesh Karnik and Qilian Liang ( $J_{x}$ appeared first in [5] and [6]) the intention was that $J_{x}$ is meant to be interpreted as (2). Regardless of the "intention," math is math and so, since $J_{x}$ in $J_{x} \subseteq[0,1]$ was originally left undefined, it is too late to say "Oh, we meant it to be defined as an IVFS (as in (2))." Hence, using the interpretation explained in [2] provides a more general definition of the IT2FS according to the mathematical definition of $J_{x} \subseteq[0,1]$. 
It is very important, however, for readers to understand (the following has not been mentioned in [2]) that all operations, methods and systems that have been developed and published about IT2 FSs are, so far, only valid in the special case when $I T 2 F S=I V F S^{1}$. Whether or not the interval type- 2 operations, methods and systems developed so far are valid, as is, or are extendable to other cases of IT2 FSs (as defined in [2]) is unknown as of early 2015. One such example is how to compute the centroid of an IT2 FS when IT2 FS $\neq$ IVFS.

In conclusion, it is believed that [2] presents a wider view on the relationship between interval-valued fuzzy sets and interval type-2 fuzzy sets. However, there is a need to explore the wider relationship between the already developed IT2 FLS theory and operations (which were developed under the special case of IT2 FS=IVFS) and how they extend when employing the other general cases of IT2 FSs presented in [2]. For example, a reviewer of this Letter asked an interesting question that we are paraphrasing as: If one begins with IT2 FSs that are not IV FSs is it possible for their join or meet not to also be IT2 FSs, e.g. could they be IV FSs?

\section{ACKNOWLEDGMENT}

The authors would like to thank the reviewers of this Letter for their suggestions and interesting questions.

\section{REFERENCES}

[1] J. Aisbett, J. T. Rickard and D. Morgenthaler, "Type-2 fuzzy sets as functions on spaces," IEEE Trans. on Fuzzy Systems, vol. 18, pp. 841844, August, 2010.

[2] H. Bustince, J. Fernandez, H. Hagras, F. Herrera, M. Pagola, and E. Barrenechea, "Interval type-2 fuzzy sets are generalization of interval-valued fuzzy sets: towards a wide view on their relationship," IEEE Trans. on Fuzzy Systems, Early Access, (DOI: 10.1109/TFUZZ.2014.2362149), 2015.

[3] N. N. Karnik and J. M. Mendel, "Operations on type-2 fuzzy sets," Fuzzy Sets and Systems, vol. 122, pp. 327-348, 2001.

[4] N. N. Karnik and J. M. Mendel, "Centroid of a type-2 fuzzy set," Information Sciences, vol. 132, pp. 195-220, 2001.

[5] N. N. Karnik, J. M. Mendel and Q. Liang, "Type-2 fuzzy logic systems," IEEE Trans. on Fuzzy Systems, vol. 7, pp. 643-658, December 1999.

[6] Q. Liang and J. M. Mendel, "Interval type-2 fuzzy logic systems: theory and design," IEEE Trans. on Fuzzy Systems, vol. 8, pp. 535-550, October 2000.

[7] J. M. Mendel, Uncertain Rule-Based Fuzzy Logic Systems: Introduction and New Directions, Prentice-Hall, Upper Saddle River, NJ, 2001.

[8] J. M. Mendel, H. Hagras and R. I. John, "Standard background material about interval type-2 fuzzy logic systems that can be used by all authors," IEEE Computational Intelligence Society, can be accessed at: cis.ieee.org/standards-committee.html, 2006.

[9] J. M. Mendel and M. R. Rajati, "On Clarifying Some Notations Used for Type-2 Fuzzy Sets as Well as Some Recommended Notational Changes," submitted for publication in Information Sciences, Dec. 2014.

\footnotetext{
${ }^{1}$ Join and meet are used for all T2 FSs. They are worked out in detail only for IT2 FS = IVFS in [6] and [7].
} 